\title{
Registering the Retinal Vasculature in Gray-scale and Color Digital Fundus Images
}

\author{
Islam A. Fouad \\ Biomedical Technology Dept. \\ Salman bin A. Aziz University \\ K.S.A., Al-Kharj
}

\author{
Fatma El-Zahraa M. Labib \\ Biomedical Engineering Dept. \\ Cairo University \\ Egypt, Cairo
}

\begin{abstract}
Some of the most common blinding conditions are caused by choroidal neovascularization (CNV). The relevant conditions include diabetic retinopathy and age-related macular degeneration. At present, the only proven modality of effective treatment is the application of laser energy to the $\mathrm{CNV}$ to cauterize the vessels. The key to effective and lasting treatment is the identification of the full extent of the CNV, cauterization of the CNV completely by accurately aiming an appropriate amount of optical energy while ensuring that healthy tissue is not cauterized. Extraction techniques must be developed to discern the retinal blood vessels tree and determine the positions of laser shots in a reference frame. After extracting the blood vessels tree \& determining the locations of the laser shots, those locations will be saved in a database. Due to the eye movement the locations of the laser shots will differ. So, registration of the translation between the reference \& the sub-sequent frame in different types of retinal images is required. This paper presents a comparison of two different methods to register blood vessels, which are a prominent retinal structure, in both gray-scale and color retinal images. The blood vessel registration is composed of two algorithms, i.e., registering the blood vessel using Normalized 2-D Correlation algorithm and Minimum Error Function algorithm. Results on various retinal images verify the effectiveness of the proposed methods.
\end{abstract}

\section{Keywords}

Retinal Image, Registration, Choroidal Neovascularization, Age-related Macular Degeneration, Correlation.

\section{INTRODUCTION}

Retinal image processing is mainly required in diagnosing and treatment of many diseases affecting the retina [1], [2], [3] and so there is a strong medical motivation for the work presented in this paper: Age-related macular degeneration (AMD) is the main reason for often severe loss and lasting decrease in visual acuity. Choroidal neovascularization $(\mathrm{CNV})$ associated with age-related macular degeneration leads to irreparable damage in structure and function of retinal tissue [4]. The therapeutic approach favors timely and complete photocoagulation of the entire peripheral retina in order to save central vision [5], [6]. Several thousand lesions are required and care is taken to avoid radiating the macula, the optic disc, the retinal blood vessels and the region between the macula and the optic disc containing the nerve fibers to the area of acute vision (the macula). For each eye, this procedure requires several thousands of laser shots. This could be achieved in several hours spaced over many treatment sessions [7]. The full extent of the affected peripheral retina must be completely coagulated while ensuring that healthy tissue is not cauterized. Despite the superiority of treatment by laser over other available methods, serious problems remain. The current rate of success of this procedure is less than $50 \%$ following one treatment session with a recurrence and or persistence rate of about 50\% [8], [9]. The latter condition requires repeating the treatment. Each treatment repetition in turn has a $50 \%$ failure rate. The recovery of vision declines with each successive treatment. Several studies indicate that incomplete treatment was associated with poorer prognosis than no treatment [10], [11], and [12]. Complete treatment can be obtained if the cauterization is done in one session. So, we assume to have a computerized system to track the retina and make the whole number of the required laser shots in on session and the system have a computer that captures the retinal images from a fundus camera, perform some image processing algorithms to these images to finally feed the positions to which the laser will be directed to a beam steering apparatus. A primary step is to design the image processing algorithms required to segment the retinal images. The goal of the segmentation step is to extract the sensitive objects in the retinal images which include: the blood vessel tree, the macula and the optic disc and the region between them. The locations to which laser will be directed are to be determined away from these sensitive objects.

Also image registration techniques are required to update the predetermined positions of laser shots according to the eye movement and saccades [10]. The registration should be achieved in real time.

In this work, comparisons of applying two different registration algorithms to both gray-scale and color retinal images are presented. The aim of this work is to detect and register the blood vessels in the retinal images accurately and automatically.

The proposed blood vessel registration algorithms are:

- Registering the retinal blood vessels using Normalized 2-D Correlation.

- Registering the retinal blood vessels using Minimum Error Function.

The registration system can be characterized by its computation time and its error probability.

\section{Determination of Locations of Laser Shots}

After extracting the blood vessels tree [13], the sensitive structure that must be avoided during laser treatment, a binary image was composed containing these objects. This image was dilated by a square structuring element of dimensions $7 \times 7$ to maintain safety margins around these sensitive areas. The locations of shots were determined and spaced in the background. These locations were stored in the database of the patient and get updated with every successive image frame. 


\section{Updating Positions of Laser Shots}

An image containing the positions to which laser will be directed is now available as a result of reference frame processing. During laser treatment, these positions should be updated according to the eye movements and saccades. Position updating process must be achieved on-line, in time period less than that of the saccadic movement, and with the maximum accuracy. Image registration algorithms can be used for this task. Image registration may be done by different algorithms, among which are used: similarity detection algorithm.

Image registration is the alignment of similar images in one or more image. The similar images are generally mis-registered by scaling, rotation, and translation. Rotation is the angular twisting of one image with respect to the other, scaling is the magnification of one image with respect to the other, and translation is the displacement of the two images in the horizontal and/or the vertical directions. Other problems can complicate the registration, such as the nonlinear distortion in one image and not in the other, or when one object is viewed from two directions. In case of retinal images, the rotational movement is known to be very small (approaching and never exceeding $5^{\circ}$ ), and the scaling of one image with respect to the other can be controlled by asking the patient to fixate his eyes, and the head is hold to the fundus camera. The translation occurs in the vertical and in the horizontal directions and as there is no retinal detachment, the retina is known to move rigidly.

When the computer time is abundant, the images can eventually be registered, usually with an excellent success rate. But if the matching must be performed in a limited time, such as during a real time task, the registration task becomes difficult if not impossible. Many methods are available to either decrease the error rate or decrease the computation time. The registration system can be characterized by its computation time and its probability of error. The computation time is defined as the amount of time for the system to perform the image registration task of one image with another image or to search and locate preselected landmarks or features within the image. The probability of error is defined as the number of incorrect matches divided by the total matches attempted. In general, decreasing the probability of error will result in an increase in the computation time, and vice versa. This tradeoff is application dependent and becomes critical in real time task.

As mentioned above, image registration can be achieved either by detecting the similarity between two images or by extracting some corresponding points and estimating the motion parameters between them as a previous techniques. In this work, the similarity detection algorithms will be applied.

\section{MATERIALS}

A block diagram of the data acquisition system is presented in Figure 1. The retinal images used in this work were acquired using a TOPCON TRC-50X fundus camera. All images collected for this work were acquired with the camera adjusted at $50^{\circ}$ mode (this corresponds to the field of the eye seen by the camera) and with the red-free illumination mode. A Sony Charge Coupled Device (CCD) video camera was attached to the eyepiece of the fundus camera to make the images collected using the fundus camera available in standard video format.

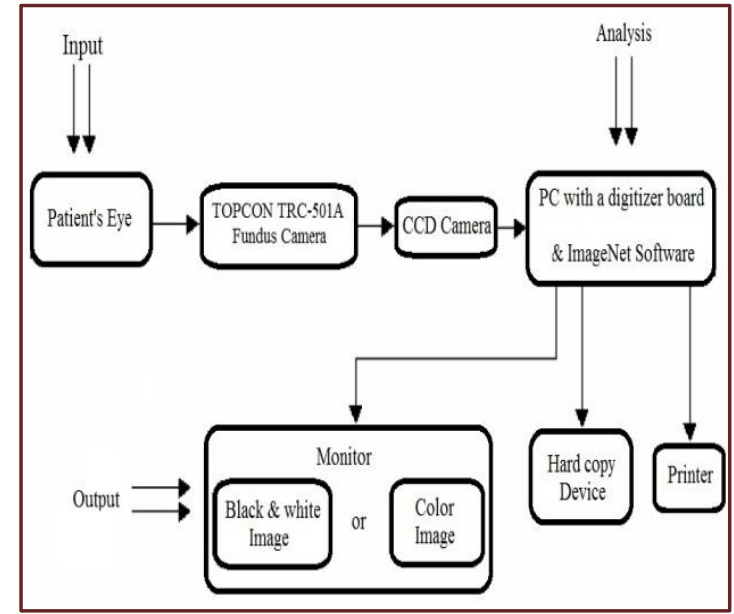

Figure 1, a block diagram of the data acquisition system

The video output from the CCD camera was interfaced to a Micron PC (866 MHz processor, 256Mbytes of RAM) through a video digitizer card. The retinal images captured by this setup may be one of the three possible types. The first type is gray level images with no injected dye which called the normal image. In this type of images, the blood vessels appear as dark objects on a bright background. The second type is gray level images with brighter blood vessels relative to a darker background. This type of images is obtained when the patient is injected with the IndoCyanine Green dye. The third type is the colored images that appear with the true colors of the retina. The images were captured to the memory of the computer system by means of a software package called Image Net2000 (version 2.03(Topcon corp. 96-98)). This package can capture either individual frames or real time video sequences. The frame size is $640 \times 480$ pixels of either colored or gray level images. The images were saved on a hard disk for further processing.

The eyes of the patient were dilated and was asked to fixate his eyes during the imaging by looking to a fixed light and holding his head to the fundus camera. Several aspects of retinal images in general and live video retinal images in particular, make automated processing difficult. First the images are highly variable. The naturally high variability of fundus images between patients is widely acknowledged. The variability of live images is especially high due to unavoidable movements, and the difficulty of providing steady illumination. For instance, the need arises to process image frames that are dim, out of focus, motion blurred, or captured with optical effects such as glare or non-uniform illumination. During photography, the skilled user quickly refocuses the camera for optimum illumination for each picture. Any images with insufficient illumination or excessive glare are simply discarded.

\section{METHODS}

\subsection{Image Registration by Similarity Detection}

Registration is inherently basic to the task of retinal tracking. When it is desired to detect the similarity between two images or to perform a mapping of two similar images, it is necessary for meaningful results to have the images registered. If the images do not differ in magnification and rotation, then the best translational fit will yield the required registration. 
Let two images, $\boldsymbol{S}$ the search area and $\boldsymbol{W}$ the window defined as shown in figure $2 . \boldsymbol{S}$ is taken as an $\boldsymbol{L} \times \boldsymbol{M}$ array of digital picture elements which may assume one of $\boldsymbol{K}$ gray levels i.e.

$$
0 \leq S(i, j) \leq K-1 \text {, }
$$

Where,

$$
1 \leq i \leq L \& 1 \leq j \leq M
$$

$W$ is considered to be an $N \times N$, ( $N$ smaller than $L, M)$ array of digital picture elements having the same gray scale range; i.e.

$$
0 \leq W(l, m) \leq K-1,
$$

Where,

$$
1 \leq l \leq L \& 1 \leq m \leq M
$$

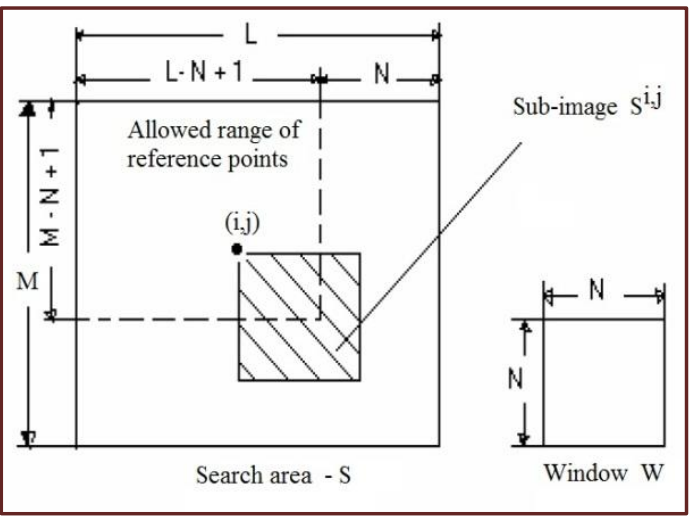

Figure 2, Search space in digital picture elements

It will be convenient to introduce a notation for $N \times N$ wholly contained sub-images.

$$
S_{N}{ }^{i, j}(l, m) \equiv S(i+l-1, j+m-1)
$$

Where,

$$
\begin{gathered}
1 \leq l, m \leq N, \\
1 \leq i \leq L-N+1, \\
1 \leq j \leq M-N+1,
\end{gathered}
$$

Each $N \times N$ sub-image can be uniquely referenced by the specification of its upper left corner's coordinates $(\boldsymbol{i}, \boldsymbol{j})$. These will be used to define reference points. It will be assumed that enough a priori information is known about the dislocation between the window and the search area so that the parameters $\boldsymbol{L}, \boldsymbol{M}$ and $\boldsymbol{N}$ may be selected with the virtual guarantee that, at registration, a complete sub-image is contained in the search area as shown in figure 2.

Therefore, the translational registration is a search over some subset of the allowed range of reference points to find a point $\left(i^{*}, j^{*}\right)$ which indicate a sub-image that is the most similar to the given window.

\subsection{Similarity Detection by Correlation}

The method most widely used for the automatic determination of translation is correlation [14], [15]. The elements of the non-normalized cross-correlation surface $R(i, j)$ are defined to be : $R(i, j)=\sum_{l=1}^{N} \sum_{m=1}^{N} W^{2}(l, m) S_{N}{ }^{i, j}(l, m)$

Where,

$$
\begin{gathered}
1 \leq i \leq L-N+1, \\
1 \leq j \leq M-N+1,
\end{gathered}
$$

In the correlation scheme, a correlation output surface such as $R(i, j)$ is searched for a maximum at $(\hat{i}, \hat{j})$. The procedure is successful if $(\hat{i}, \hat{\mathrm{j}})$ and $\left(i^{*}, j^{*}\right)$ are equivalent. As a counterexample, however, consider the non-normalized cross correlation of the previous equation even in the ideal case where $\mathrm{W}$ exactly matches some sub-image; i.e. $W=S_{N}{ }^{i^{*}, j^{*}}$. Then

$$
R\left(i^{*}, j^{*}\right)=\sum_{l=1}^{N} \sum_{m=1}^{N} W^{2}(l, m)
$$

Also for this ideal case, consider the non-matching point $(\hat{\imath}, \hat{j})$ where

$S_{N}^{\hat{i}, \hat{j}}(l, m)=\max _{l, m} W(l, m)=W_{N} \quad$ for all $\quad l, m$, Clearly,

$$
R(\hat{i}, \hat{j})=W_{N} \sum_{l=1}^{N} \sum_{m=1}^{N} W(l, m) \geq R\left(i^{*}, j^{*}\right) .
$$

Therefore, even in the ideal case, a search for a maximum over $R(i, j)$ does not necessarily yield the registration point. Normalization is therefore necessary in even the simplest of cases.

$$
R_{N}^{2}(i, j)=\frac{\left(\sum_{l=1}^{N} \sum_{m=1}^{N} W(l, m) S_{N}{ }^{i, j}(l, m)\right)^{2}}{\left[\sum_{l=1}^{N} \sum_{m=1}^{N} W^{2}(l, m)\right]\left[\sum_{l=1}^{N} \sum_{m=1}^{N} S^{2}{ }_{N}{ }^{i, j}(l, m)\right]},
$$

$$
1 \leq i \leq L-N+1, \quad 1 \leq i \leq L-N+1,
$$

The choice of a similarity detection algorithm should be justified by its probability for error and its computational complexity rather than by tradition or expediency. Perhaps the two reasons, which are generally given for using the correlation method, are that: 1) correlation appears to be a natural solution for the mean-square-error criteria [15]; and 2) analog-optical method implements correlation easily [15].

However, there is no guarantee for any method that a solution is correct or unique. There seems to be, therefore, no adequate justification for the use of correlation to solve all digital registration problems.

\subsection{Similarity Detection using Minimum Error Function}

For a particular reference point $(i, j)$, there are $N^{2}$ points of the sub-image $S_{N}^{i, j}$, which may be compared with the $N^{2}$ corresponding points in $W$. Each set of points for comparison (e.g., $\left.\left[S_{N}^{i, j}(l, m), W(l, m)\right]\right)$ will be called a windowing pair.

Here, a search over each of the $(L-N+1)(M-N+1)$ reference points is performed. Non normalized or normalized measures for evaluating the error between windowing pairs may be defined as:

$$
\varepsilon^{\prime}(i, j, l, m) \equiv\left|S_{N}^{i, j}(l, m)-W(l, m)\right|,
$$

and

$$
\begin{aligned}
& \varepsilon(i, j, l, m) \equiv\left|S_{N}^{i, j}(l, m)-\hat{S}(i, j)-W(l, m)+\hat{W}\right|, \\
& \text { Where, } \quad \hat{W} \equiv \frac{1}{N^{2}} \sum_{l=1}^{N} \sum_{m=1}^{N} W(l, m),
\end{aligned}
$$


and

$$
\hat{S}(i, j) \equiv \frac{1}{N^{2}} \sum \sum S_{N}^{i, j}(l, m) .
$$

Unlike the correlation method cited previously, in the ideal case, where $W=S_{N}{ }^{i *} j^{*}$, a minimum of zero is guaranteed for the non-normalized case, i.e., for

$$
\begin{gathered}
\|E(i, j)\| \equiv \sum_{l=1}^{N} \sum_{m=1}^{N}\left|S_{N}^{i, j}(l, m)-W(l, m)\right|, \\
0=\left\|E\left(i^{*}, j^{*}\right)\right\| \leq\|E(i, j)\| .
\end{gathered}
$$

Thus, in this ideal case no normalization is necessary. But, the normalized case is usually used because it gives accurate results than the non-normalized case because it deals with relative intensity values. The point of minimum response (minimum error) will represent the point of matching.

\section{RESULTS AND DISCUSSIONS}

In this section, the results of the detection of motion parameters will be listed and explored for the previously described registration algorithms.

\subsection{Results of Similarity Detection by Correlation}

The results of registering two normal, ICG and color images, using the previously described algorithm of calculating the normalized 2-dimensional cross-correlation, are shown in figures 3, 4 and 5 respectively.

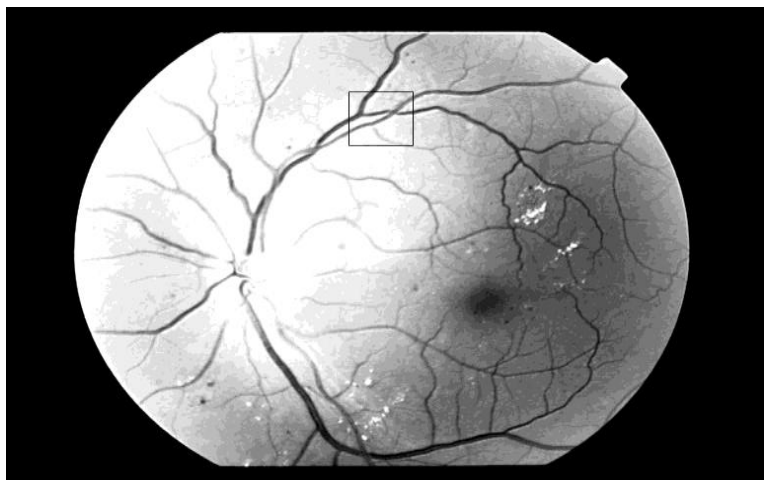

a. The window chosen in the Reference Normal Image for matching

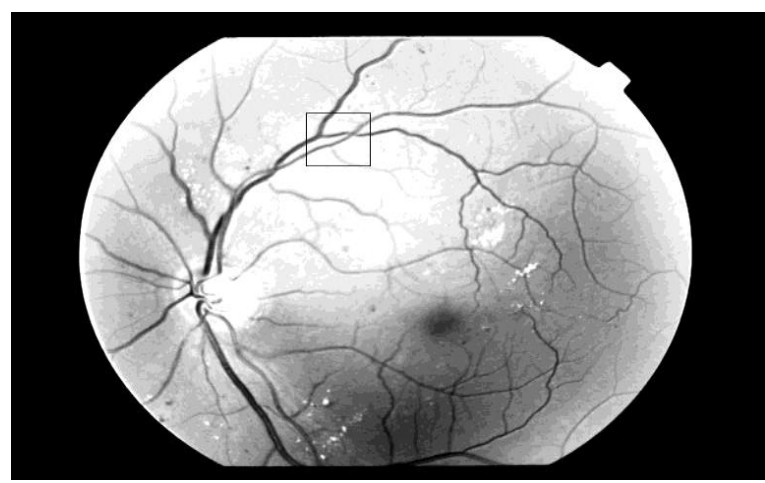

b. The position of the window after matching using the normalized correlation

Figure 3, Results of applying the "2-D Cross-Correlation" to register two normal images.

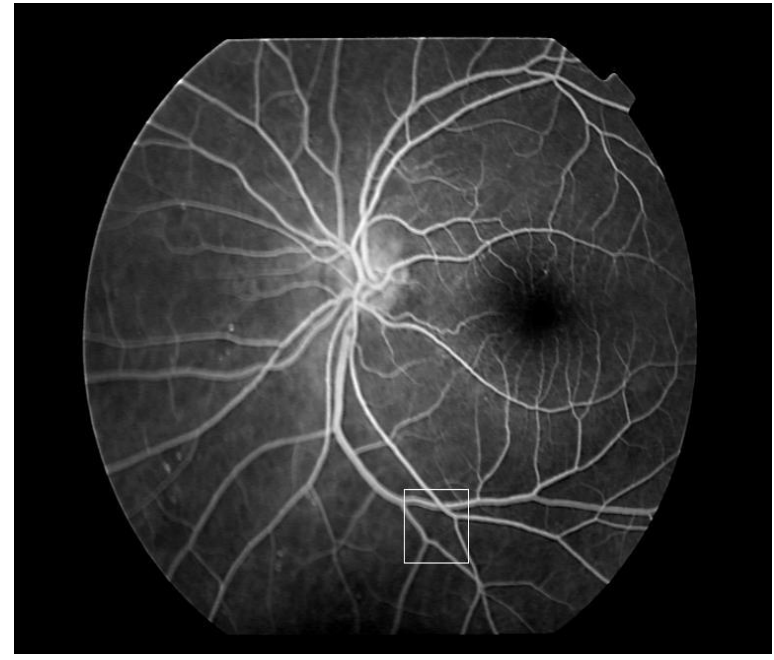

a. The window chosen in the Reference ICG Image for matching

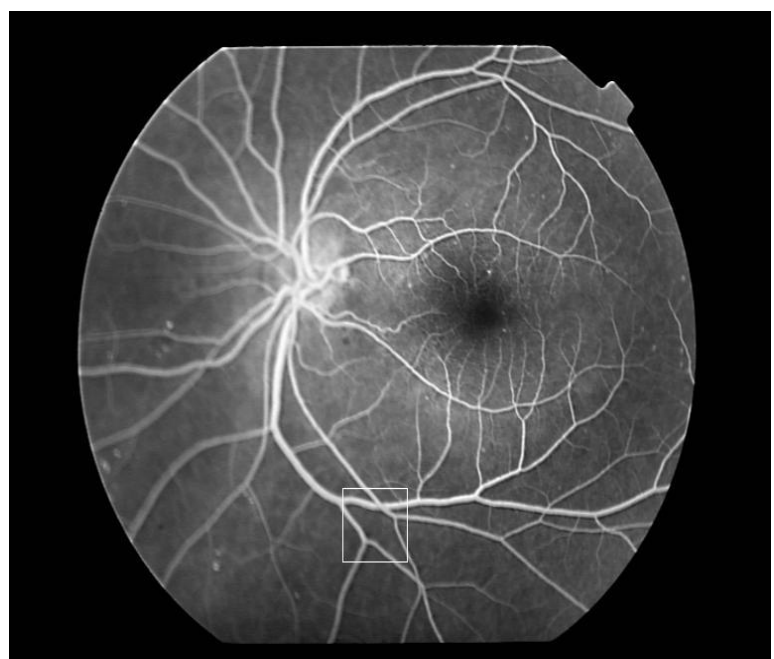

b. The position of the window after matching using the normalized correlation

Figure 4, Results of applying the "2-D Cross-Correlation" to register two ICG images.

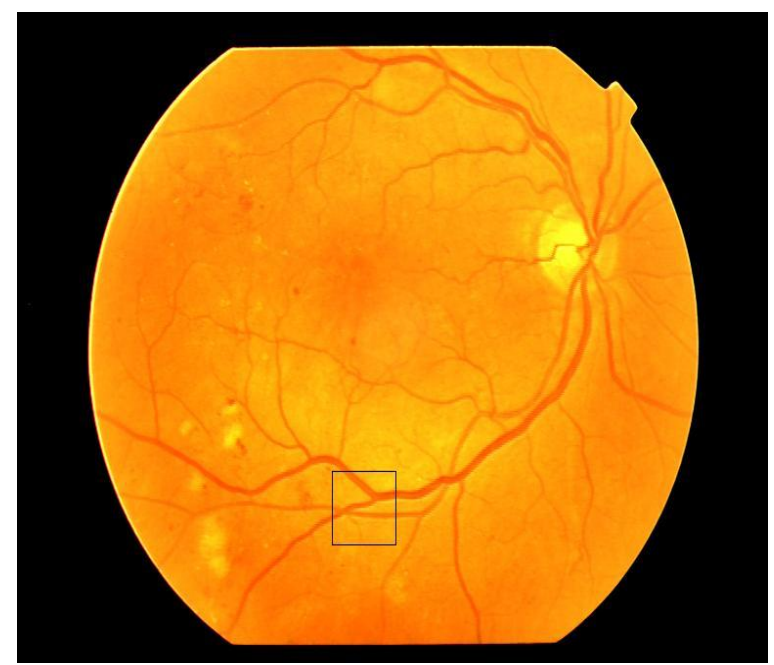

a. The window chosen in the Reference Color Image for matching 


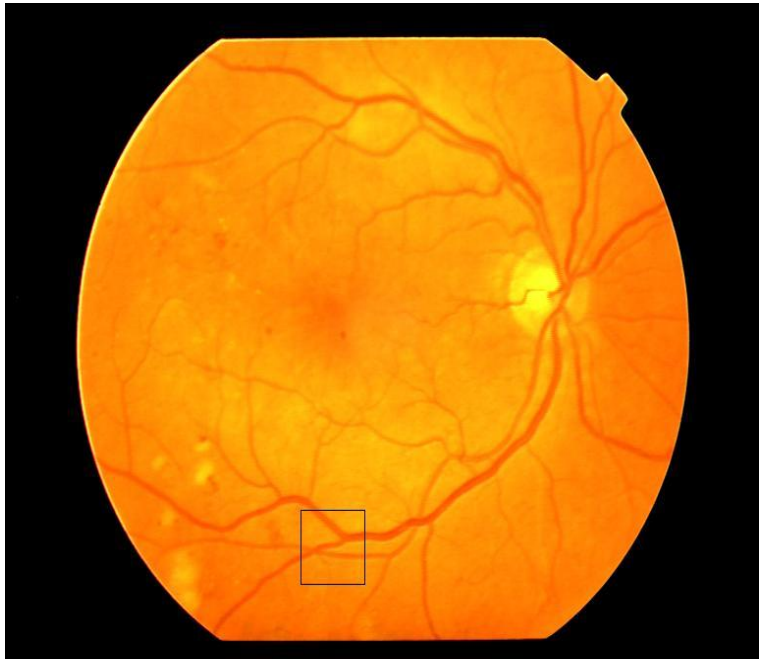

b. The position of the window after matching using the normalized correlation

Figure 5, Results of applying the "2-D Cross-Correlation" to register two color images.

Working with color image as in figure 5 , it was separated into its three components $(\mathrm{R}, \mathrm{G} \& \mathrm{~B})$ and apply the pre-described registration algorithm to any of those components. In this work, it was dealt with second frame (the GREEN frame) as it was found that it has good contrast and gives best results as shown.

It was found that, applying this technique is not suitable for retinal tracking, as it depends on the features of the selected window on the image.

\subsection{Results of Similarity Detection by Calculating Minimum Error Function}

The results of registering two normal, ICG and color images, using the previously described algorithm of calculating the minimum error function, are shown in figures 6,7 and 8 respectively.

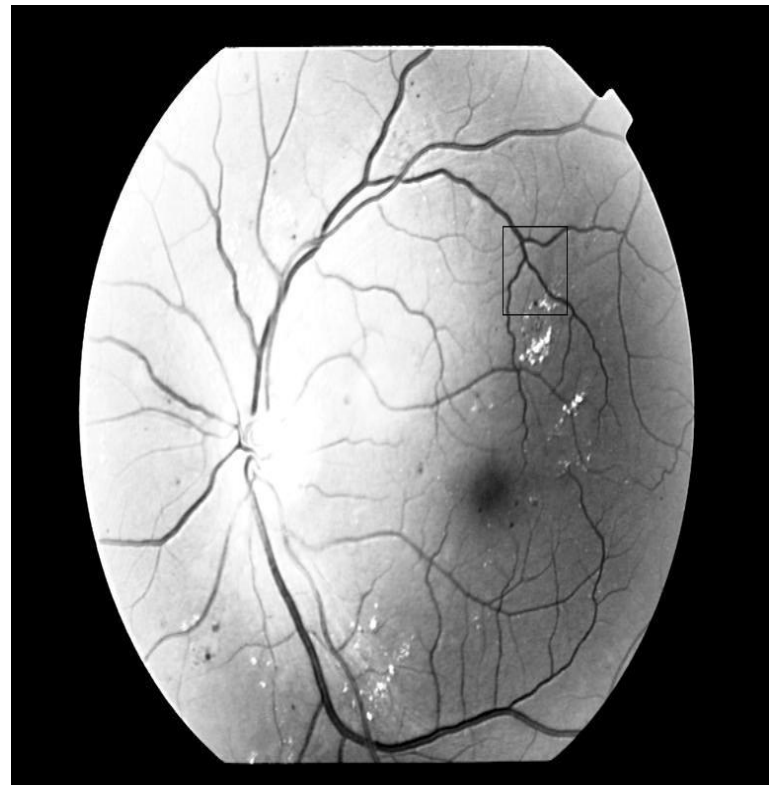

a. The window chosen in the Reference Normal Image for matching

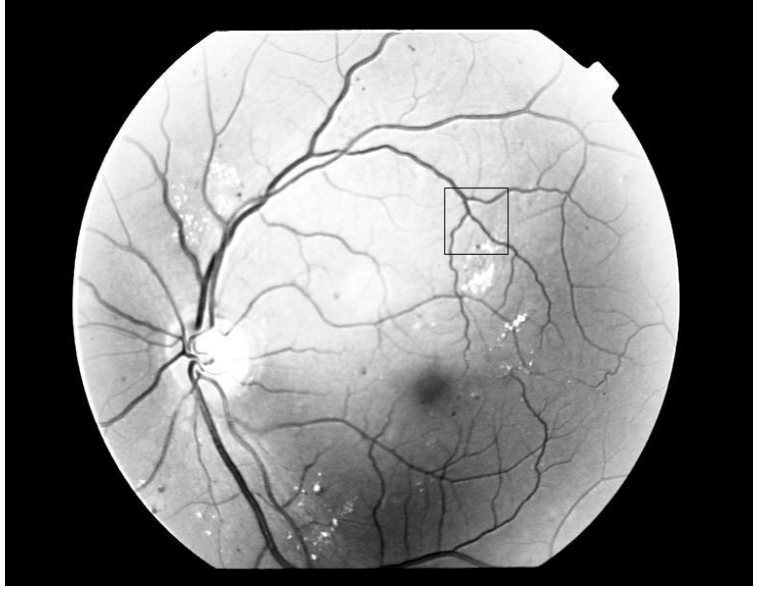

b. The position of the window after matching using the Minimum Error Function

Figure 6, Results of applying the "Minimum Error Function" to register two normal images.

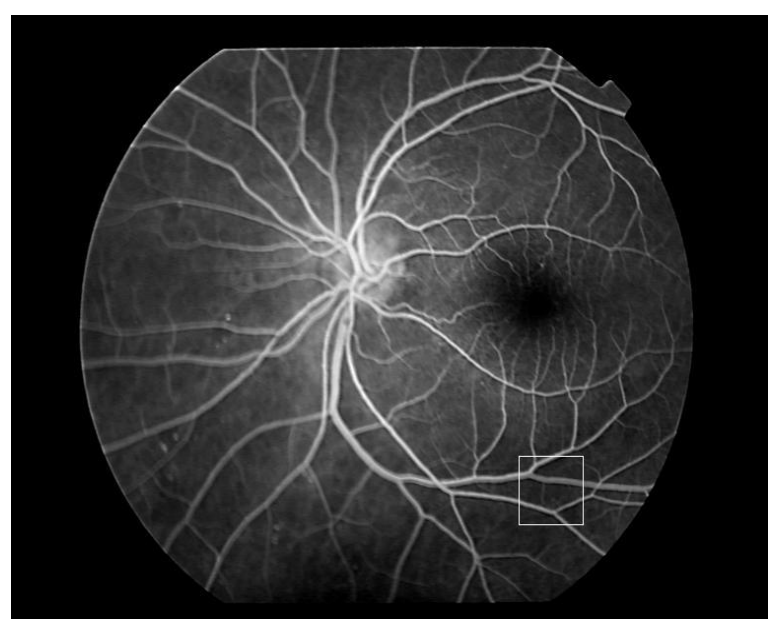

a. The window chosen in the Reference ICG Image for matching

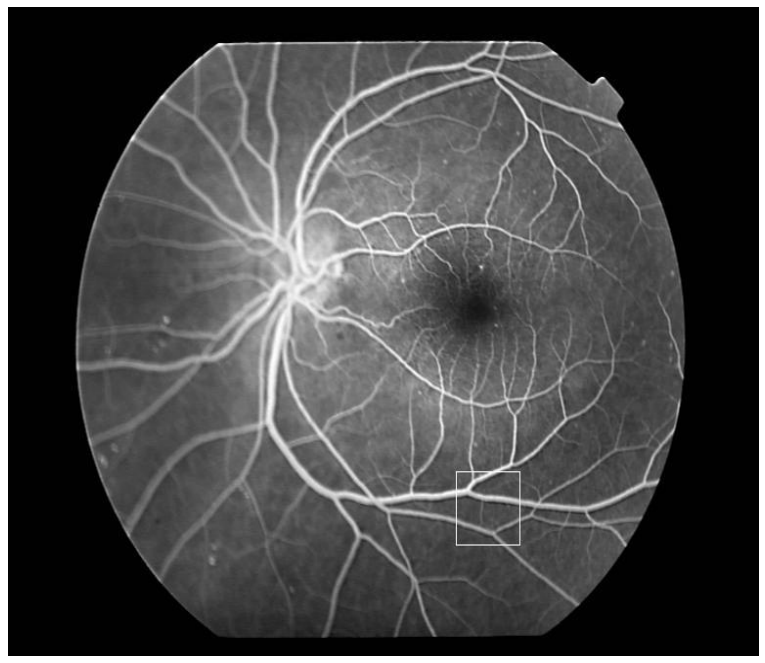

b. The position of the window after matching using the Minimum Error Function

Figure 7, Results of applying the "Minimum Error Function" to register two ICG images. 


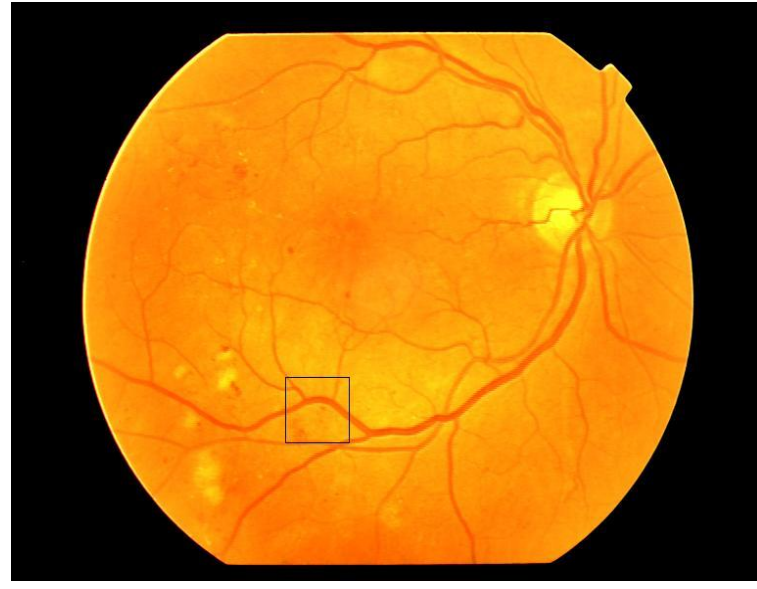

a. The window chosen in the Reference Color Image for matching

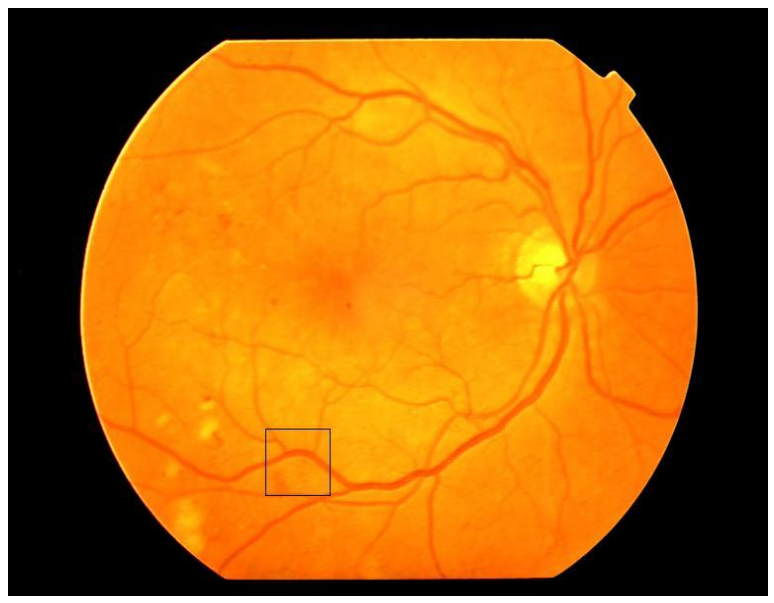

b. The position of the window after matching using the Minimum Error Function

Figure 8, Results of applying the "Minimum Error Function" to register two color images.

It was mentioned previously that the color image was separated into its three components (Red, Green, and Blue) and apply the pre-described registration algorithm to any of those components. In this work, it was dealt with the second frame (the GREEN frame) as it was found that it has good contrast and gives best results as shown in figure 7 .

It was found that applying this technique is not suitable for on-line retinal tracking, as it depends on the features of the selected window on the image $\&$ also it takes too much time.

Table 1. A brief comparison among the two presented algorithms for the purpose of registering the retinal images

\begin{tabular}{|c|c|c|c|}
\hline \multirow{2}{*}{$\begin{array}{c}\text { TYPE } \\
\text { OF } \\
\text { IMAGE }\end{array}$} & \multicolumn{3}{|c|}{ Normalized 2-D Correlation } \\
\cline { 2 - 4 } & Accuracy & Atomicity & $\begin{array}{c}\text { Execution time } \\
\text { (Sec.) }\end{array}$ \\
\hline Normal & High & Low & 2.844 \\
\hline ICG & High & Low & 2.891 \\
\hline
\end{tabular}

\begin{tabular}{|c|c|c|c|}
\hline Color & High & Low & 3.281 \\
\hline $\begin{array}{c}\text { TYPE } \\
\text { OF } \\
\text { IMAGE }\end{array}$ & \multicolumn{3}{|c|}{ Minimum Error Function } \\
\cline { 2 - 4 } & Accuracy & Atomicity & $\begin{array}{c}\text { Execution time } \\
\text { (Sec.) }\end{array}$ \\
\hline Normal & High & Low & 5.574 \\
\hline ICG & High & Low & 5.884 \\
\hline Color & High & Low & 7.111 \\
\hline
\end{tabular}

According to table.1, the algorithms can be reliable to be used in retinal image registration since it can be assumed that the translation is the only existing motion parameter. Rotation is minimal if present and can be compensated for. The scaling between images can be controlled.

The presented methods have a high accuracy on images with various kinds of noise. Those applicable methods can correctly register all the three types of retinal images without any human intervention.

The results of registering the retinal image using the correlation method and the minimum error function are good. In these two methods, the window $W$ used in matching must be chosen properly to obtain good results as errors in registration appear when using a window with no obvious features.

It should be noted that the processing time for the registering of the three various types of retinal images, was lower than 10 sec. (processor: Intel(R) Core (TM) i5 - $2.27 \mathrm{G} \mathrm{Hz}$ ), rendering the technique a valuable tool for retinal tracking application.

\section{CONCLUSION}

Recall that the purpose of the retinal tracking process is to help CNV associated patients to stop their visual loss. This is achieved by automatic extraction \& registration of blood vessels tree.

In this study, two different techniques for automatic registration of blood vessels tree in different types of retinal images were demonstrated. The results obtained so far suggest that: applying extraction techniques on gray-scale images is so much easier than applying those on color images as in the color image the algorithm is applied to the three $R, G \& B$ color image components, and so the time taken \& computational effort in the color images making the process harder. On the other hand, the results showed on the sample retinal images give almost the same vessels appearance \& background clearance on both cases. So, it was found that it's important to convert the color retinal image into a gray-scale image as a pre-processing step to get reasonable results in a minimum time.

Some possible points for future work are proposed based on the work done as follows:

1. Applying more image registration algorithms as :

- Methods of retinal images mosaicing.

- Methods of Spatial Transformation.

- Methods of Mutual Information. 
2. The movement of the eye will be tracked to obtain the speed of the retina movement and hence calculating the taken time and the corresponding pixel shifts in the retinal images.

3. As the retina is part of a spherical surface, the retinal images will be handled as three dimensional images. The results of both the proposed algorithm and that will be obtained from the three dimensional images will be compared. Then, the better algorithm will be considering in the system implementation.

4. The work done is to be realized in the future through the implementation of the hardware.

\section{REFERENCES}

[1] B. JL..1986. Photo physical Processes in Recent Medical Laser Developments. Lasers Med Sci., vol. 1, pp.47-66.

[2] B. GM..1986. Lasers in Medicine and Surgery, JAMA, vol. 256, pp. 900-907.

[3] T. M. Clark, W. R. Freeman, and M. H. Goldbaum.1992. Digital Overlay of Flourescein Angiograms and Fundus Images for Treatment of Subretinal Neovascularization, J. Retinal, Vitreous Diseases, vol. 2, pp. 118-126.

[4] S. B. Bressler, N. M. Bressler, S. L. Fine, A. Hillis, R. P. Murphy, R. J. Olk, and A. Patz.1982. Natural course of choroidal neovascular membranes within the foveal avascular zone in senile macular degeneration. Amer.J. Ophthalmol., vol. 93, pp. 157-163.

[5] Kay A. Ball.1995. Lasers, The Perioperative Challenge. 2nd edition, Mobsy-Year Book, Inc.

[6] Bruce M. Achuer, Vectoria M. Vander Kam and Michael W. Berns.1992. Lasers in Plastic Surgery and Dermatology. Thieme Medical Publishers, Inc. New York.
[7] S.F. Barrett, M.R. Jerath, H. Grady Rylander III and Ashley J. Welch.1994. Digital Tracking and Control of Retinal Images. Optical Engineering, 33, no.1, pp.150159.

[8] N.M. Bressler, S.B. Bressler, and E.S. Gragoudas.1987. Clincal characteristics of choroidal neovascular membranes. Arch. Ophthalmol., 105, pp. 209-213

[9] P.N. Monahan, K. A. Gitter, and G. Cohen.1993. Evaluation of Persistence of Subretinal Neovascular Membranes Using Digitized Angiographic Analysis. Retina-J. Retinal, Vitreous Diseases, 13, no. 3, pp. 196201.

[10] S. Fine.1988. Observations Following Laser Treatment for Choroidal Neovascularization. Arch. Ophthalm., 106, pp. 1524-1525.

[11] N. M. Bressler, S.B. Bressler, and E.S. Gragoudas.1987. Clincal characteristics of choroidal neovascular membranes. Arch. Ophthalmol., vol. 105, pp. 209-213.

[12] P. N. Monahan, K. A. Gitter, and G. Cohen.1993. Evaluation of Persistence of Subretinal Neovascular Membranes Using Digitized Angiographic Analysis. Retina-J. Retinal, Vitreous Diseases, vol. 13, pp. 196201.

[13] Fouad, I. A..2015. A Comparative Study of Retinal Vasculature Extraction in Digital Fundus Images. Journal of Biomedical Engineering and Technology, 3(1), 1-7.

[14] B. Peli, R.A. Augliere and G.T. Timberlake.1987. Feature-Based Registration for Retinal Images. IEEE Trans. Med. Imaging, vol. MI-6, no. 3, pp.272-278.

[15] D.I. Barnea and H.F. Silverman.1972. A Class of Algorithms for Fast Digital Image Registration. IEEE Transaction on Computers, vol.C-21, no.2, pp.179-186. 